\title{
Genetic variation in CYB5R3 is associated with methemoglobin levels in preterm infants receiving nitric oxide therapy
}

\author{
Tyson D. Fuller', Cassandra N. Spracklen², Kelli K. Ryckman², Lindsey A. Knake', Tamara D. Busch', Allison M. Momany', \\ Jeffrey C. Murray ${ }^{1}$ and John M. Dagle ${ }^{1}$
}

BACKGROUND: In recent years, increasing numbers of preterm infants have been exposed to inhaled nitric oxide (iNO). This population has decreased methemoglobin (MetHb) reductase activity in their erythrocytes, which may increase the risk of MetHb toxicity. We sought to determine if genetic factors are associated with the observed variance in MetHb levels. METHODS: A population of 127 preterm infants was genotyped for five single-nucleotide polymorphisms (SNPs) in the CYBSA and CYB5R3 genes. iNO dose and levels of MetHb were obtained by chart abstraction. ANOVA was performed to identify genetic associations with MetHb levels.

RESULTS: An association was found between the heterozygous genotype (GA) of rs916321 in the CYB5R3 gene and the mean of the first recorded MetHb levels in Caucasian infants ( $P$ $=0.01)$. This result remained significant after adjustment for the iNO dose $(P=0.009)$, gender $(P=0.03)$, multiple gestation ( $P=$ $0.03)$, birth weight $(P=0.02)$, and gestational age $(P=0.02)$. No significant associations were found with the other SNPs.

CONCLUSION: We demonstrate a novel genetic association with neonatal MetHb levels. Identification of genetic risk factors may be useful in determining which preterm infants are most at risk of developing MetHb toxicity with the use of iNO.

N itric oxide (NO) is naturally produced in the vascular endothelium by the enzyme NO synthase (1). NO enters the pulmonary vascular smooth muscle cells either by simple diffusion or through extracellular formation of SNO-L-cysteine which enters through the L-type amino acid transporter (2). There, it stimulates soluble guanylate cyclase, which produces the intracellular second messenger cyclic guanosine monophosphate, a mediator in the vasodilatory pathway $(1,3)$. When $\mathrm{NO}$ enters the blood stream, it quickly reacts with hemoglobin to form methemoglobin (MetHb), and as a result, the hemoglobin is no longer able to bind oxygen $(1,4,5)$. The concentration of MetHb in the blood under normal conditions is typically less than $2 \%$ of all hemoglobin (6). When levels of MetHb in the blood reach elevated concentrations, MetHb toxicity can occur (6-9).

Preterm infants have lower than normal levels of MetHb reductase, the enzyme responsible for converting MetHb back to functional hemoglobin, which puts them at increased risk for developing MetHb toxicity (9). At the same time, however, preterm infants are being treated with inhaled NO (iNO) as therapy to prevent chronic lung disease $(1,8-10)$. In order to prevent MetHb toxicity, MetHb levels are frequently measured while the preterm infant is receiving iNO therapy $(8,9)$.

CYB5A (cytochrome B5 type A) and CYB5R3 (cytochrome B5 reductase 3) are genes that encode proteins with MetHb reductase activity, each of which have two major splice variants. A soluble form of these proteins in the erythrocytes is responsible for reducing MetHb back into functional hemoglobin. Several studies have demonstrated that methemoglobinemia is associated with genetic variations in both CYB5A and CYB5R3, resulting in decreased enzyme activity (7,11-15).

Although genetic influences may play a role in a preterm infant's susceptibility to elevated MetHb levels following iNO exposure, this association has not yet been studied. Thus, the objective of this study was to evaluate the association between two single-nucleotide polymorphisms (SNPs) in CYB5A and three SNPs in CYB5R3, two genes known to influence MetHb levels, and MetHb levels in preterm infants receiving iNO. This information will give us a better understanding of the genetic influence on MetHb levels and may help to determine more appropriate iNO administration and monitoring of MetHb levels in this vulnerable population.

\section{RESULTS}

Characteristics of our primary study population can be seen in Table 1. Of the 109 Caucasian preterm infants receiving iNO therapy, the majority of the infants were male, non-Hispanic, of singleton gestation, born weighing $<1,000 \mathrm{~g}$, and born at $\leq 28$ wk gestation. There was no statistical difference in MetHb levels with respect to gender, multiple gestation, birth weight, gestational age, or ethnicity.

The average starting dose of iNO in this cohort was 12.60 $\mathrm{ppm}$. The first MetHb level was selected for analysis as this was assumed to be the closest to the baseline MetHb levels for the infants. The time interval from starting iNO to checking the first MetHb value varied between 1 and 24h. Using a Pearson's 
Table 1. Characteristics of Caucasian study participants

\begin{tabular}{|c|c|c|c|}
\hline Covariate & $N(\%)$ & Methemoglobin value & $P^{a}$ \\
\hline \multicolumn{4}{|l|}{ Gender } \\
\hline Male & $68(62.4)$ & $0.837(0.361)$ & 0.98 \\
\hline Female & $41(37.6)$ & $0.839(0.356)$ & \\
\hline \multicolumn{4}{|l|}{ Multiple gestation } \\
\hline No & $86(78.9)$ & $0.843(0.348)$ & 0.74 \\
\hline Yes & $23(21.1)$ & $0.817(0.399)$ & \\
\hline \multicolumn{4}{|l|}{ Birth weight (g) } \\
\hline$<1,000$ & $67(61.5)$ & $0.820(0.353)$ & 0.84 \\
\hline $1,000-1,499$ & $10(9.2)$ & $0.872(0.366)$ & \\
\hline $1,500-2,499$ & $15(13.8)$ & $0.907(0.485)$ & \\
\hline$\geq 2,500$ & $17(15.6)$ & $0.846(0.266)$ & \\
\hline \multicolumn{4}{|l|}{ Gestational age (wk) } \\
\hline$\leq 28$ & $73(68.9)$ & $0.809(0.347)$ & 0.37 \\
\hline $29-32$ & $9(8.5)$ & $0.911(0.404)$ & \\
\hline $33-36$ & $24(22.6)$ & $0.912(0.394)$ & \\
\hline \multicolumn{4}{|l|}{ Ethnicity } \\
\hline Non-Hispanic & $103(94.5)$ & $0.840(0.355)$ & 0.96 \\
\hline Hispanic or Latino & $6(5.5)$ & $0.834(0.435)$ & \\
\hline
\end{tabular}

correlation analysis, we found an association between the first iNO dose and the first metHb value measured $(P=0.04)$.

The maximum iNO dose and corresponding MetHb levels were also found to be correlated $(P=0.0006)$. Finally, we found no evidence of a consistent increase in MetHb levels with duration of iNO exposure.

Table 2 compares the mean of the first recorded MetHb level with each genotype for the five analyzed SNPs. Infants heterozygous (GA) for SNP rs916321 in the CYB5R3 gene have significantly lower MetHb levels than either homozygote $(P=0.01)$ (Figure 1). This association remained significant after adjustment for the first iNO level $(P=0.009)$, gender $(P=0.03)$, multiple gestation $(P=0.03)$, birth weight $(P=0.02)$, and gestational age $(P=0.02)$. The association also remained significant when rs916321 variants were compared with the average metHb levels in the first week of iNO exposure (adjusting for iNO dose). Although the difference found in the MetHb levels between rs916321 variants was small, it was statistically significant and may show a larger effect in interactions with other genetic or environmental risk factors. No significant associations were found between the first measured MetHb level and the other four genotyped SNPs.

Additional analyses show that the highest recorded MetHb level measured was also associated with rs916321 genotype

Table 2. Methemoglobin means and SDs for evaluated SNPs among Caucasians only

\begin{tabular}{|c|c|c|c|c|c|c|c|c|}
\hline \multirow[b]{2}{*}{ SNP } & \multirow[b]{2}{*}{$N$} & \multirow{2}{*}{$\begin{array}{c}\text { MetHb level, } \\
\text { mean (SD) }\end{array}$} & \multirow[b]{2}{*}{$P$ value $^{\mathrm{a}}$} & \multicolumn{5}{|c|}{ Adjusted $P$ value ${ }^{a}$} \\
\hline & & & & First NO level & Gender & Multiple gestation & Birth weight & Gestational age \\
\hline \multicolumn{9}{|c|}{ rs8096066 } \\
\hline AG & 46 & $0.893(0.42)$ & & & & & & \\
\hline GG & 3 & $0.733(0.31)$ & & & & & & \\
\hline GG & 48 & $0.836(0.33)$ & 0.68 & 0.04 & 0.83 & 0.57 & 0.80 & 0.81 \\
\hline GT & 50 & $0.897(0.41)$ & & & & & & \\
\hline $\mathrm{TT}$ & 11 & $0.845(0.26)$ & & & & & & \\
\hline \multicolumn{9}{|c|}{ rs6002829 } \\
\hline $\mathrm{CC}$ & 34 & $0.878(0.32)$ & 0.95 & 0.05 & 0.98 & 0.72 & 0.97 & 0.96 \\
\hline \multicolumn{9}{|c|}{ rs916321 } \\
\hline GG & 46 & $0.934(0.39)$ & 0.01 & 0.009 & 0.03 & 0.03 & 0.02 & 0.02 \\
\hline GA & 47 & $0.752(0.31)$ & & & & & & \\
\hline AA & 15 & $1.006(0.30)$ & & & & & & \\
\hline \multicolumn{9}{|c|}{ rs2285138 } \\
\hline $\mathrm{TT}$ & 34 & $0.845(0.32)$ & 0.68 & 0.08 & 0.84 & 0.60 & 0.82 & 0.83 \\
\hline $\mathrm{TC}$ & 55 & $0.855(0.38)$ & & & & & & \\
\hline CC & 20 & $0.927(0.34)$ & & & & & & \\
\hline
\end{tabular}

MetHb, methemoglobin; NO, nitric oxide; SNP, single-nucleotide polymorphism.

a $P$ values from ANOVA testing difference between mean methemoglobin values among the three different genotypes for each SNP. 


\section{Articles | Fuller et al.}

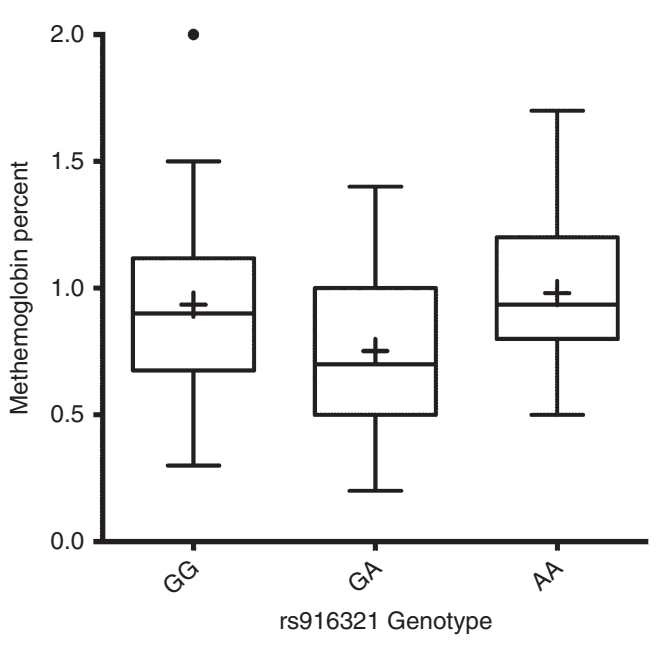

Figure 1. MetHb level by genotype for the SNP rs916321. The mean (represented by the plus sign) of the first recorded MetHb level was plotted by genotype for the SNP rs916321. As a group, heterozygous infants (GA) had significantly lower MetHb levels than homozygous infants (GG or $\mathrm{AA} ; P=0.01$ ). The center line within each box represents the median, while the bottom and top horizontal lines that close the box represent the first and third quartiles, respectively. The whiskers that extend from the box represent the 10th-90th percentile of distribution. The black circle above the GG box and whisker represents a MetHb value that is beyond the 90th percentile. However, this value does not exceed beyond 3 SDs of the mean and, as such, is not considered to be an outlier. MetHb, methemoglobin; SNP, single-nucleotide polymorphism.

$(P=0.0035$, adjusted for iNO dose received prior to measurement). The average of all the MetHb levels abstracted from the medical charts was also associated with rs916321 genotype $(P=0.02$, adjusted for average abstracted iNO dose).

Results from a secondary analysis evaluating the association between the first measured MetHb level and the five SNPs in all infants in the study population (not stratified by race) can be found in Supplementary Table S1 online. Results in this analysis were similar to the results for Caucasian infants only.

\section{DISCUSSION}

iNO at doses of 10-80 ppm has been shown to improve oxygenation in term and near-term infants without significant toxicity or effects on systemic circulation $(9,10)$. However, the use of iNO can lead to excessive oxidative stress and toxic levels of MetHb (methemoglobinemia) $(7,8)$. To prevent clinically significant methemoglobinemia, infants receiving iNO have $\mathrm{MetHb}$ levels checked frequently; however, some centers are questioning the utility of this practice $(8,9)$.

Our selection of candidate genes was driven by the known biology of congenital methemoglobinemia. This condition is caused by deficiencies in the cytochrome b5 reductase enzyme, with two distinct, previously described forms of methemoglobinemia (I and II) $(7,11,13,15)$. Type I is a benign form caused by a deficiency in the soluble erythrocyte form of the enzyme, typically due to amino acid substitutions that result in protein instability. In most cases, type I methemoglobinemia can be corrected with methylene blue or ascorbic acid and riboflavin, and patients with type I methemoglobinemia are expected to have a normal life expectancy $(7,13)$. Type II is a more severe form of methemoglobinemia that affects all cells, resulting in developmental defects, neurological disabilities, and early mortality $(13,14)$. Studies have shown different genetic polymorphisms linked to type I and type II methemoglobinemia (12-14). Recent studies have also found genetic polymorphisms associated with atypical type II disease phenotypes $(14,16)$. Thus, investigation of common polymorphisms present in these genes was expected to provide information regarding MetHb levels.

We have shown one SNP (rs916321) to have an association with MetHb levels of preterm infants receiving iNO. The MetHb levels were decreased in the GA heterozygote individuals compared to the AA or GG homozygote subjects. Results obtained using the AA subjects should be interpreted in the context of the small number of minor allele homozygotes in our population $(n=15)$. Our findings of heterozygote infants having lower MetHb levels than either homozygote may also be an example of positive heterosis. This is most likely due to increased allelic diversity in heterozygotes which is expected to increase the biochemical and molecular network versatility within these individuals (17). This would then increase the possible ways for heterozygotes to overcome different environmental stresses such as iNO administration.

It is important to note that rs916321 is a marker for a specific region of the genome and is likely not the etiologic cause of variations in MetHb values. Using data present in the 1000 genomes project website, it was determined that rs916321 is in linkage disequilibrium (i.e., genetically linked) with many other common variants such as rs3761461, rs5758824, and rs11705171. These three SNPs all have $r^{2}$ values of 1.0, suggesting complete linkage disequilibrium and are all in areas likely to be involved in gene regulation. rs3761461 is located in a potential CCTC-binding factor binding site region and is also located near a possible FOS-like antigen 2 binding site. This SNP is also in a region of the genome with high DNase hypersensitivity, suggesting that this area may be important for gene regulation. rs5758824 has been shown to be in a region likely regulating gene activity in lung fibroblasts. Finally, rs11705171 is near a site which is suggested to be highly involved in gene regulation in several cell types including blood vessel, blood, skin, and muscle cells. More detailed analysis of this region of CYB5R3 and the specific regulatory elements impacting CYB5R3 transcription could provide important information regarding the biological variability that we have found in MetHb levels in preterm infants.

To the best of our knowledge, this is the first study to look for genetic associations with MetHb levels in preterm infants receiving iNO. In this study, we looked at genes known to play a role in MetHb reduction, as they would be more likely to show association with MetHb levels. Strengths of this study include a well-defined phenotype in a clinically significant population. In addition, analyzing a Caucasian cohort 
simplifies interpretation of our data by decreasing the possible contribution of glucose-6-phosphate dehydrogenase deficiency-induced hemolysis to MetHb level. Limitations of the study include the sample size and small number of SNPs studied. Increasing the overall sample size and number of SNPs analyzed would help increase power and give us better coverage of the genes to increase informativity. In addition, our retrospective analysis used data obtained during routine clinical care, thus the duration of iNO exposure varied greatly among subjects. Our study population was also selected from a convenience sample of infants already enrolled in a biobank at the University of Iowa Children's Hospital, and our findings may not be generalizable to other populations.

Finally, MetHb reduction is a very complex process and, as a result, several genetic and environmental factors likely influence overall MetHb levels. For the purposes of this study, we chose to analyze the genes we believed to be the most influential on MetHb levels. Future studies will be important in the discovery of other additional environmental and genetic risk factors that may have an even larger effect on MetHb levels.

In conclusion, using a candidate gene analysis, we found that rs916321 in the CYB5R3 gene is associated with MetHb levels in preterm infants receiving iNO. Replication of this SNP in a larger, independent population is required to further confirm the association between this polymorphism and MetHb levels. Ultimately, associations such as the one presented here may prove useful in determining the most appropriate iNO dose administered to preterm infants and may reduce or eliminate the need to frequently monitor MetHb levels.

\section{METHODS}

\section{Sample Population}

This study population was chosen from an existing cohort of infants admitted to the neonatal intensive care unit at the University of Iowa Children's Hospital between 2000 and 2013. Infants in the initial cohort were recruited as part of an initiative to create a research biobank of biologic material (blood and/or buccal swabs) from infants for subsequent use in investigations into the genetic contributions of neonatal conditions (18-20). Parental informed consent was obtained for inclusion in this biobank. Within this cohort, we identified a subgroup of 127 preterm infants ( $<37 \mathrm{wk}$ gestation) for our analysis who received iNO secondary to hypoxemic respiratory failure despite aggressive medical management. All preterm infants in the initial cohort who received iNO were included. None of the infants in the study had their iNO dose lowered because of concerns of MetHb toxicity. Term infants were excluded from the study. Demographic and clinical data were abstracted from the infant's medical records. All study protocols and procedures were approved by the University of Iowa Institutional Review Board.

\section{Candidate Gene/SNP Selection}

Candidate genes were selected based on a review of the literature and biological plausibility. Genes known to be involved in the process of $\mathrm{MetHb}$ reduction were specifically chosen for analysis. SNPs within each gene were selected for analysis based on haplotype blocks that would give the best coverage of each gene using the fewest SNPs possible. This strategy allowed for $89 \%$ coverage of CYB5A and $53 \%$ coverage of $C Y B 5 R 3$. In order to increase the information obtained at each locus, a minor allele frequency cutoff of 0.2 was used to ensure that an adequate number of individuals from the sample population would be carriers of the minor allele. Using this method, five SNPs from two genes were selected for genotyping (CYB5A: rs8096066, rs7238784, and rs6002829; CYB5R3: rs916321 andrs2285138).

\section{Genotyping}

DNA used in the analysis was extracted from discarded umbilical cord blood or buccal swabs using standard protocols. The TaqMan genotyping system (Applied Biosystems, Foster City, CA) was used to determine allelic variation for each SNP. Genotyping reactions were completed using dried DNA samples in 384-well plates, per a previously described protocol that is similar to the manufacturer's recommendation (21). The Sequence Detection Systems software (version 2.2) preloaded on the ABI $7900 \mathrm{HT}$ was used for the allele scoring of each SNP.

\section{Statistical Analysis}

All statistical analyses were performed using SAS 9.3 (SAS Institute, Cary, NC). ANOVA was performed to assess for differences in the first measured MetHb level and each demographic or clinical variable. ANOVA was also performed to test for differences in the first measured MetHb level and each of the SNPs. Infants whose first $\mathrm{MetHb}$ value was more than 3 SDs away from the mean for each genotype were excluded from the analysis $(n=2)$. Covariates were evaluated and included gender (male/female), whether the infant was a twin or triplet (multiple gestation, yes/no), birth weight in grams (continuous), and gestational age in weeks (continuous). Due to the potential for racial heterogeneity in genetics, the primary analyses only included the 109 Caucasian infants. Additional analyses were also performed including all of the available subjects $(n=127)$; these were additionally adjusted for race (white/nonwhite). All SNPs were found to be in Hardy-Weinberg equilibrium using Fisher exact tests. $\mathrm{MetHb}$ levels were found to be normally distributed.

\section{SUPPLEMENTARY MATERIAL}

Supplementary material is linked to the online version of the paper at http:// www.nature.com/pr

\section{STATEMENT OF FINANCIAL SUPPORT}

This study was supported by March of Dimes (White Plains, NY) FY11-261 and FY13-19.

Disclosure: The authors have no financial ties to disclose.

\section{REFERENCES}

1. Porta NF, Steinhorn RH. Pulmonary vasodilator therapy in the NICU: inhaled nitric oxide, sildenafil, and other pulmonary vasodilating agents. Clin Perinatol 2012;39:149-64.

2. Brahmajothi MV, Mason SN, Whorton AR, McMahon TJ, Auten RL. Transport rather than diffusion-dependent route for nitric oxide gas activity in alveolar epithelium. Free Radic Biol Med 2010;49:294-300.

3. Steinbicker AU, Liu H, Jiramongkolchai $\mathrm{K}$, et al. Nitric oxide regulates pulmonary vascular smooth muscle cell expression of the inducible cAMP early repressor gene. Nitric Oxide 2011;25:294-302.

4. Vega-Villa K, Pluta R, Lonser R, Woo S. Quantitative systems pharmacology model of NO metabolome and methemoglobin following long-term infusion of sodium nitrite in humans. CPT Pharmacometrics Syst Pharmacol 2013;2:e60.

5. Goetz BI, Shields HW, Basu S, et al. An electron paramagnetic resonance study of the affinity of nitrite for methemoglobin. Nitric Oxide 2010;22:149-54.

6. Manassaram DM, Backer LC, Messing R, Fleming LE, Luke B, Monteilh CP. Nitrates in drinking water and methemoglobin levels in pregnancy: a longitudinal study. Environ Health 2010;9:60.

7. Elahian F, Sepehrizadeh Z, Moghimi B, Mirzaei SA. Human cytochrome b5 reductase: structure, function, and potential applications. Crit Rev Biotechnol 2014;34:134-43.

8. Hamon I, Gauthier-Moulinier H, Grelet-Dessioux E, Storme L, Fresson J, Hascoet JM. Methaemoglobinaemia risk factors with inhaled nitric oxide therapy in newborn infants. Acta Paediatr 2010;99:1467-73.

9. Peliowski A; Canadian Paediatric Society, Fetus and Newborn Committee. Inhaled nitric oxide use in newborns. Paediatr Child Health 2012;17: 95-100. 


\section{Articles | Fuller et al.}

10. Ballard RA, Truog WE, Cnaan A, et al.; NO CLD Study Group. Inhaled nitric oxide in preterm infants undergoing mechanical ventilation. $\mathrm{N}$ Engl J Med 2006;355:343-53.

11. Choury D, Leroux A, Kaplan JC. Membrane-bound cytochrome b5 reductase (methemoglobin reductase) in human erythrocytes. Study in normal and methemoglobinemic subjects. J Clin Invest 1981;67:149-55.

12. Hamirani YS, Franklin W, Grifka RG, Stainback RF. Methemoglobinemia in a young man. Tex Heart Inst J 2008;35:76-7.

13. Lorenzo FRt, Phillips JD, Nussenzveig R, et al. Molecular basis of two novel mutations found in type I methemoglobinemia. Blood Cells Mol Dis 2011;46:277-81.

14. Percy MJ, Barnes C, Crighton G, Leventer RJ, Wynn R, Lappin TR. Methemoglobin reductase deficiency: novel mutation is associated with a disease phenotype of intermediate severity. J Pediatr Hematol Oncol 2012;34: 457-60.

15. Tanishima K, Fukuda N, Takeshita M, Takizawa Y, Kitamura T, Yoneyama Y. Automated determination of red cell methaemoglobin reductase activity by a continuous-flow system for screening hereditary methaemoglobinaemia. J Clin Pathol 1979;32:584-9.
16. Ewenczyk C, Leroux A, Roubergue A, et al. Recessive hereditary methaemoglobinaemia, type II: delineation of the clinical spectrum. Brain 2008;131(Pt 3):760-1.

17. Andorf S, Gartner T, Steinfath M, Witucka-Wall H, Altmann T, Repsilber D. Towards systems biology of heterosis: a hypothesis about molecular network structure applied for the Arabidopsis metabolome. EURASIP J Bioinform Syst Biol 2009:147157.

18. Abu-Maziad A, Schaa K, Bell EF, et al. Role of polymorphic variants as genetic modulators of infection in neonatal sepsis. Pediatr Res 2010;68:323-9.

19. Dagle JM, Lepp NT, Cooper ME, et al. Determination of genetic predisposition to patent ductus arteriosus in preterm infants. Pediatrics 2009;123:1116-23.

20. Mohamed S, Schaa K, Cooper ME, et al. Genetic contributions to the development of retinopathy of prematurity. Pediatr Res 2009;65: 193-7.

21. Ehn NL, Cooper ME, Orr K, et al. Evaluation of fetal and maternal genetic variation in the progesterone receptor gene for contributions to preterm birth. Pediatr Res 2007;62:630-5. 